\title{
Exploring Multi-Modal Communication Approach for Young Children with Spinal Muscular Atrophy (SMA)
}

\author{
Sheng Miao \\ School of Data Science and \\ Software Engineering \\ Qingdao University \\ miao.sh7@live.com
}

\author{
Ziying Tang, Jinjuan Heidi Feng \\ Department of Computer and \\ Information Sciences \\ Towson University \\ ztang, jfeng@ towson.edu
}

\author{
Amanda Jozkowski \\ Department of Occupational \\ Therapy and Occupational sciences \\ Towson University \\ ajozkowski@towson.edu
}

\begin{abstract}
Spinal Muscular Atrophy (SMA) is a rare genetic disease affecting approximately 1 in 10,000 babies, yet it is the number 1 genetic killer of infants and toddlers. Individuals with SMA, especially the most serve type I, can face great challenges in communication, environment control, and learning knowledge. Since most children with type I SMA have extremely limit muscular functionality, they cannot use regular interactive devices. In this study, we propose a multi-modal communication approach and explore various sensors and switches for SMA users. Specifically, we propose a light-weight and wireless microcontroller to process electric signals from sensors and switches. It can be paired and used in any devices which supports Bluetooth. Moreover, an interactive game and a three-phase pilot study are designed for assessing usability of various input devices.
\end{abstract}

\section{Introduction}

Spinal Muscular Atrophy (SMA) is an autosomal recessive neurodegenerative disease characterized by the degeneration of spinal cord motor neurons, atrophy of skeletal muscles, and generalized weakness [1]. Most of the nerve cells that control muscles are located in the spinal cord which accounts for the word spinal in the name of the disease. SMA is muscular because its primary effect is on muscles, which do not receive signals from these nerve cells. Atrophy is the medical term for getting smaller, which is what generally happens to muscles when they are not active [2]. The incidence of SMA is about one in every 6000 to 10,000 livebirths with a carrier frequency of one in $50[3,4]$. Nowadays, SMA affects more than 750,000 patients worldwide and it is the number one genetic killer of infants and toddlers [5]. Unfortunately, there was no effective medical treatment until Nusinersen was approved by the FDA in December 2016 [6]. However, the cost of this treatment is humongous: the first year of treatment will cost $\$ 750,000$ and $\$ 375,000$ per year after that [7]. Moreover, previous research has shown that the median total cost for patients with SMA were $\$ 83,652$ over an average of 6.9 years and the median cost for patients with early diagnosis was $\$ 167,921$ [8]. Individuals with SMA and their families have heavy medical burden.

Previous research [9] has shown that approximately 80 percent of individuals with SMA fall into type I which is the most severe category. Type I SMA is also called Werdnig-Hoffmann disease; it leads infants to severe muscle weakness before 6 months of age. These young children never achieve the ability to sit independently when placed on their own; therefore, they are completely dependent on others for all aspects of their care and participation. Furthermore, most of them have extremely limited muscle functionality; they may only use several fingers with very little pressure. As a result, they cannot use regular interactive devices such as a mouse, a keyboard or even a touch screen. Since most patients with type I SMA cannot walk or talk, they may face significant challenges from the following three aspects. First, they have difficulties communicating efficiently with others, so it is hard to express their thoughts and ask for help. Second, individuals with SMA cannot control the environment around them, for example they may have problems tuning on the light in living room or turning off the television. The third challenge is that children with type I SMA have limited resources when learning about the world, as they cannot interact with computer systems effectively. Therefore, solving these challenges is very critical and helpful. Since these young children desire to interact and engage with the world, it is a crucial time for intelligence development. How to provide communication assistance becomes a fairly vital research question for this special user group.

With the rapid development of information technology and various sensors, research on assistive living and communication systems have become very active in the past few decades. [10] has proposed an 
assistive system to improve independence for people with autism spectrum disorder and [11, 12] have done some research on improving social, academic or organization skills. Furthermore, there is also research for other populations such as deaf children [13] and older adults [14]. Although different methods and technologies have been proposed, none of them can be applied for our target users: the users in previous research have strong muscular functionality while our target user, young children with type I SMA, cannot use traditional interactive methods and require special switches and sensors due to their disabilities. And this study can be extended to help other populations such as people with regular muscular atrophy and amyotrophic lateral sclerosis.

In this paper, our research focus is to study what are the alternative interacting method for young children with type I SMA. Specifically, we propose a multi-modal interaction approach which employs multiple interactive sensors to improve SMA users capability of interacting with computer based virtual environment. Our goal is to help users performing accurate input and control computer systems in the future. This is the foundation for high efficient communication and environment control, so that to decrease living dependent. The rest of paper is organized as follow: chapter 2 introduces the background and related work in the field of assistive systems for users with disability; chapter 3 presents our multiple-modal interaction system. A brief pilot study is explored in chapter 4 , and chapter 5 concludes the paper.

\section{Related work}

Existing research on young children with Spinal Muscular Atrophy (SMA) is quite limited. No directly related work was found from the assistive technology and accessible computing field that examines how to use information technology to help these children communicate with other people or control their environment. Previous research on SMA [15] has shown that type I SMA will cause the most serious weakness on movement and language ability; however, individuals have intact attentiveness and intellect. There are some studies that have examined multiple systems for users with variable disabilities which are similar to SMA.

Switch-based devices are effective and easy to use for users to input with. Typically, switches are mechanically actuated, may come in a variety of sizes and materials, and may be placed in various locations including near the hands, elbows, shoulders, feet, or head [16]. Most researchers use the simplest switches that support only one function and can be mapped to a single keyboard key when connected to a PC. [17] introduces a one-key system with a simple switch and [18] presents a customized system with wireless embedded switches for teenagers with Duchenne Muscular Dystrophy. Another input category is accessible touch and gesture. [19] explores touch screen use and tapping performance for people with motor impairments. The usability of multi-touch gestures on a smartphone touch screen is evaluated in [20]. Besides these two input groups, pressuresensitive input can also be used. [21] applies pressure input pad to power wheelchair armrests, which enables multiple inputs and force feedback joystick control for power wheelchairs is studied by [22].

With the rapid development of information technology, some research turns to non-contact inputs, like eye-tracking and brainwave measurement. Eyetracking systems can measure the point of a gaze and the motion of the eye ball relative to the head; brainwave measurements monitors the electrical activities of the brain and predicts the intention of users. [23] introduces eye tracking methodology and [24] presents eye tracking in human-computer interaction and usability research.

However, this previous research cannot be applied to our target users because type I SMA patients possessed significantly low physical capabilities. Most switches are too strong for them to push down and they cannot even keep their hands-on switches. A gesture touchpad requires precise control of hand movements, which is impossible for our target users. And there is no directly related research to assess the usability of pressure-sensitive sensors for type I SMA patients. Moreover, since eye tracking and brainwave measurement techniques require the users to be in a specific position, for example in front of an eye tracker, which means the applicability is extremely limited.

\section{Multi-modal interaction approach}

The focus of our research is to explore how to provide an accessible and responsive interactive way to allow young children with SMA to easily communicate and control their environment. In addition, considering the technology background of our user population, our solution should be customizable and usable, which means it should not take more than a few minutes to set up and it can also easily be understood and modified by families who are not technically trained. In the following sections, we explain our approaches and findings in detail. 


\subsection{User requirements}

Our target user is very young children, approximately 18 months-5 years, with type I Spinal Muscular Atrophy (SMA). In order to understand their requirements and special needs and find out the most appropriate method for them to express their thoughts, we have consulted with occupational therapists for SMA kids, interviewed SMA users caregiver and parents, and discussed with Human-Computer-Interaction expert who has worked with disability kids for years. After these investigation, we think summarize user requirements can be summarized into three parts:

- Interactive feedback. Real-time feedback is the first priority requirement since children in this age can be impatient and easily frustrated. Delayed response from the system may cause emotional reactions and decreased usability, especially for kids like our users.

- Kids-friendly user interfaces. Well-designed user interface is another very important requirement. Young children are easily attracted by their favorite character, such as super heroes and adorable animals. So those characters can help our users navigate and interact with the system. Moreover, subdued color can relax children and they can adapt to the system quickly.

- Cost-efficient and customizable. To be able to widely support our target users, we require the proposed system employs inexpensive sensors and application platforms to achieve our economic goal. Additionally, every single user is a special individual, which means user interface should be customizable and multiple design solutions should be provided.

\subsection{Wearable interactive devices}

As explained before, the traditional interacting approach based on mouse, keyboard, and touch screen are no longer suitable in our study. Alternative methods such as gesture, motion or voice based interaction cannot be applied either. As mot of type I SMA users cannot talk and have only limited little finger/hands movements. Therefore, we propose using wearable interactive devices to capture users limited movements. By doing so, if a virtual communication and control system can be provided, SMA users can utilize these wearable devices to express their thoughts and communicate with others through the virtual system. After discussing with health professional and HCI experts, we believe the wearable interactive device should have the following unique characteristics:

- Customizable (i.e., can be modified/adapted for others with SMA and similar disorders)

- Affordable/accessible to people in the SMA community

- Usable (does not take more than 5 minutes to set up and can easily be understood and modified by families who are not technically trained)

- Durable

- Responsive/sensitive

- Accurate

- Position independent. Rather than having to craft additional custom positioning solutions which bring the user to the input device, we bring the device to the user in a position which is comfortable and safe for long term regular use

- Improves quality of life and meets the needs of very young children (approximately 18 months-5 years), for whom very few therapeutic products currently exist

- Allows for maximal functional movement patterns (i.e., palms of hands are not obstructed so they can still be used for play)

Our wearable interactive device basically consists of three parts: sensor/switch, microcontroller, and virtual application. Virtual Application (VA) is the software which converts users needs to a specific description like an image or some texts. For example, if a user wants to drink water, he/she can choose the water image in the VA. Virtual application depends on the purpose of interaction and is not the focus of this paper. Here we would like to emphasis on the other two parts which can be connected to any VAs.

The purpose of microcontroller is to manage and connect sensors and switches. In our system, we employ a Feather $32 \mathrm{u} 4$ single board microcontroller, which is manufactured by Adafruit. This board is Arduino-compatible and integrated with Bluetooth Low Energy (BLE). Arduino is an open-source electronics platform based on both its hardware and software. We can use the Arduino board and software library to read various analog or digital inputs from switches and sensors. BLE is a new low-power, $2.4 \mathrm{GHz}$ spectrum wireless protocol and can be compatible with iOS, Android, Mac OS X and Windows 8+. The core chip of 
this board is ATmega32u 4 clocking at $8 \mathrm{MHz}$ and at $3.3 \mathrm{~V}$ logic. With $32 \mathrm{~K}$ of flash and $2 \mathrm{~K}$ of RAM, it can act like a mouse or keyboard easily and effectively. Moreover, micro USB and battery charging ports are both provided in this board, and it is extremely convenient for portable projects. In this system, a 3.7V Lithium polymer battery is used as the power supply.

To connect sensors and switches with the microcontroller, a $1 / 4$ size breadboard and multiple $10 \mathrm{~K}$ ohm pull-down resistors are used for the circuit. There are two channels designed in the breadboard for various switches and sensors so they can work synchronously.

Sensors and switches are used in the wearable interactive devices to capture users movements and convert them into meaningful signals to control VA. The sensors and switches we consider to adapt to use in the interactive device need to be not only sensitive enough to capture tiny finger/hand movements, but also comfortable, light-weight and portable, as it is designed for long term regular use. After careful research, we propose to include the following four types of sensor/switch: micro light switch, flex sensor, EMG based muscle sensor, and stretch sensor.

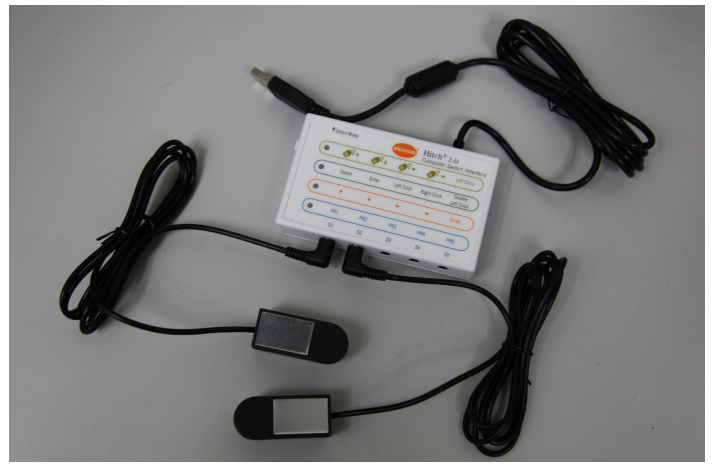

Figure 1. Micro light switches

3.2.1. Micro light switch This kind of switch allows the user to trigger it with a feather light touch. For example, the one manufactured by AbleNet only requires $0.4 \mathrm{oz}(10 \mathrm{~g})$ of force, which meets the requirement for our targeted users. With the feedback of auditory click and tactile, users can receive instant interaction results. Furthermore, the material of this switch is ABS, which is skin-friendly and provides easy mounting. 1 is an example of a micro light switch used in our system. We also include an adaptor which converts an analog signal to a digital signal and connects to a computer via a USB as shown in the figure. The switches can be mounted on wheelchair, standing on a frame, and even bed. These kinds of switches are highly effective for users who still maintain some functions on fingers. Moreover, these micro light switches can be connected to microcontrollers and paired to any devices that support Bluetooth.

3.2.2. Flex sensor Flex sensor is also manufactured by Adafruit, which can detect flexing or bending in one direction. They are popularized by being used in the Nintendo PowerGlove as a gaming interface. Basically, this sensor is a resistor that changes value based on how much it is flexed. The resistance is about $25 \mathrm{Kohm}$ when it is unflexed, while the number will rise to about $100 \mathrm{Kohm}$ when flexed all the way. Both directional bendings can be traced and will produce electric signals. 2 shows the flex sensor wiring with a microcontroller. Two different sizes of flex sensors can be used to adapt to users of different ages and in various joints. These kinds of sensors can also be used for users who have functionality on their wrist, ankle, or elbow.

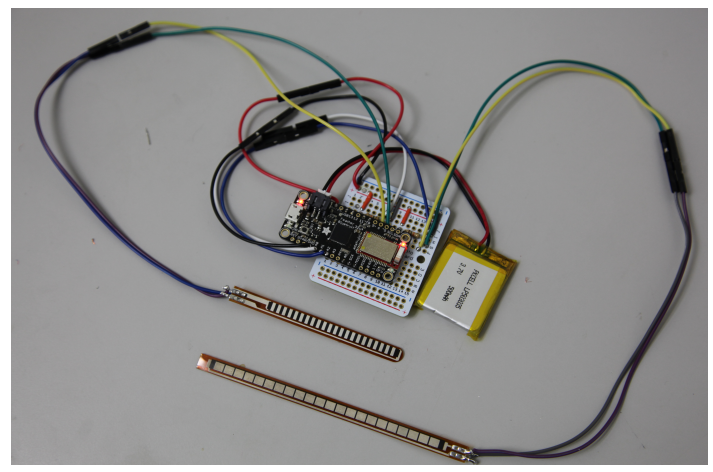

Figure 2. Flex sensors

3.2.3. Electromyography (EMG) based muscle sensor MyoWare Muscle Sensor can be used with Muscle Sensor Surface EMG Electrodes to track electromyograph and reasoning muscle contraction. Electromyography is an electrodiagnostic medical technique used for evaluating and recording the electrical activity produced by skeletal muscles. With this sensor, users can use muscle contractions as inputs to control the interaction. If a user with SMA can contract muscles on thigh or sup brows, an EMG sensor can capture these tiny movement. 3 shows the EMG sensor connected with a microcontroller and battery.

3.2.4. Stretch Sensor Stretch sensor is based on conductive yarn material which can conduct electricity. The conductive yarn spun from a stainless-steel 


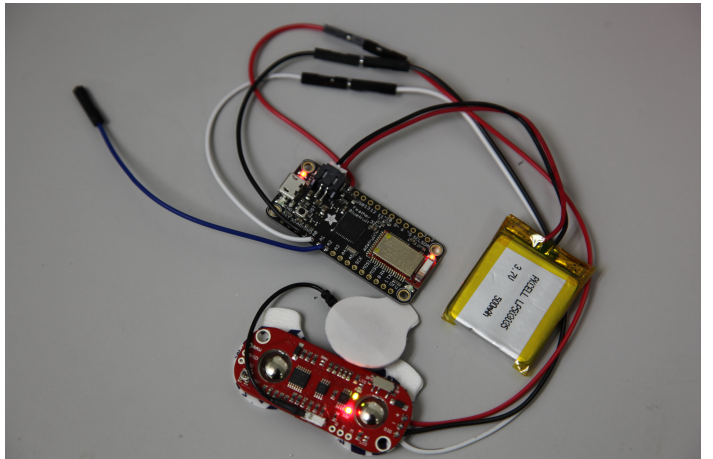

Figure 3. EMG based muscle sensor

polyester fiber blends and provides extremely pliable and soft touch. It also performs like an alternative resistor and the resistance will change when the yarn is stretched. In this study, conductive yarn is woven as a finger glove and the user can bend fingers to produce electric signals. 4 shows the sensors and circuit.

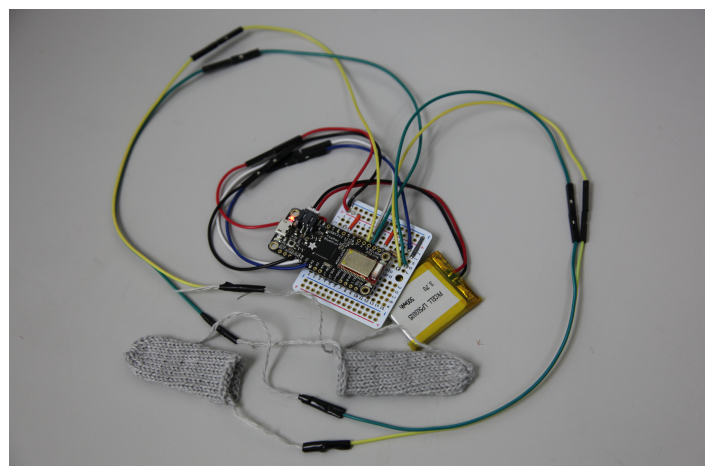

Figure 4. Stretch sensors

\section{Experiment design}

In order to evaluate the effectiveness of the proposed wearable interactive devices when SMA kids use them, we have implemented a very simple virtual selection application and designed a three phase pilot user study, considering our target users age and physical conditions. In the following section, we explain our user study in detail.

\subsection{Participant}

The participant of our user study is a three-year-old boy with type I SMA. Like most children with the same medical condition, he is fully intact cognitively but unable to function independently in many domains. He uses a tracheotomy tube and ventilator to breathe, and requires assistance to sit and to hold his head up.
Due to extreme muscle weakness, our user wears an exoskeleton and other assistive/mobility devices to help him utilize his minimal movements. Furthermore, he requires 24-hour supervision to monitor his vital signs. Our participant is cognitively very healthy. He is very friendly, active and eager to communicate with others. $\mathrm{He}$ has some limited finger and hand movements which can be well controlled. Unintentionally movement may happen but not very often. Our participant has interacted with computer systems with the help from his caregivers and parents.

We also include the caregivers and parents of the participant into our user study. The participants caregivers include one occupational therapist, one physical therapist and one language therapist. All of them can provide us important feedbacks from health professional point of view. In addition, our users parents evaluate the proposed devices from another perspective. Both of his parents are with higheducation and high-income. It is worth noticing that our participants father holds high degree of electric engineer, so they have no problem understanding the devices and their suggestions about our system are highly valuable.

\subsection{Interactive game}

Our virtual selection game has simple graphic interfaces, instant audio feedback, kids friendly color, and is very easy to understand. The game has adopts our users favorite canton character, Minion, and asks him to select the Minion icon from some bear icons. The game has four different levels, in which different numbers of icons are displayed. User needs to use two input devices for switching the cursor and selecting the target. User can only access the next level when choosing the correct minion icon. The switches and sensors used for input are binding to a key on a regular keyboard. 5 is a screenshot of the interactive selection game.

When a user plays the interactive selection game, every action from the user is recorded and delivered to Healthcare Professionals (HPs). 6 is a screenshot that shows the operational data from a user. With the help from HPs, the following attributes are identified and logged when users play the interactive game:

- Total time of current level: It represents the staying time of a user in current level. Long duration normally means the user cannot use the current sensor very well or cannot control the interaction device well.

- Number of false selections: When a user selects a bear icon which is incorrect answer, a counter 
will self-increase until the user completes current level. It reveals how possible the user trigger sensors unconscious.

- Total clicks of current level: It is a counter that records the number of actions taken by a user. This represents the efficiency of interaction system and designed user interface layout.

- Time taken to select answer: This attribute records the time between a user moves cursor to correct answer and triggers selecting operation.

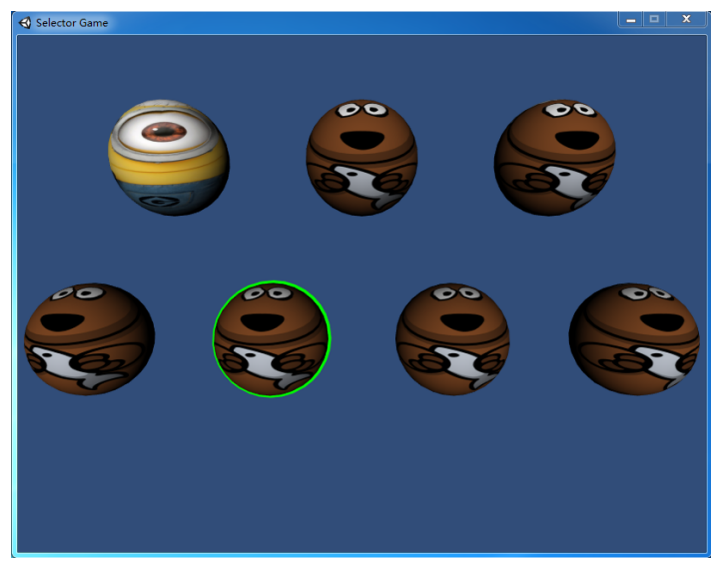

Figure 5. Interactive game

All the above attributes are recorded automatically when a user interacts with the selection game. Those data can be used to evaluate the usability of the wearable interactive device. In addition, we could also study how the layout and numbers of icons displayed affect user interaction through this game. This analysis result is very helpful when design our communication and environment control virtual application.

\subsection{Pilot study}

We have designed a three-phase pilot user study to evaluate how our participant users different kinds of wearable interactive devices. The first phase is to identify muscle functionality of the user and to interview parents; the second phase is to implement input sensors and help users understand the test system; the last phase focuses on user evaluation and feedback collection. In the following sections, we explain each phase in detail.

4.3.1. First phase During this phase, the major task is to observe participants, since every individual of the target user group is unique and in different functionalities. For example, some children may use

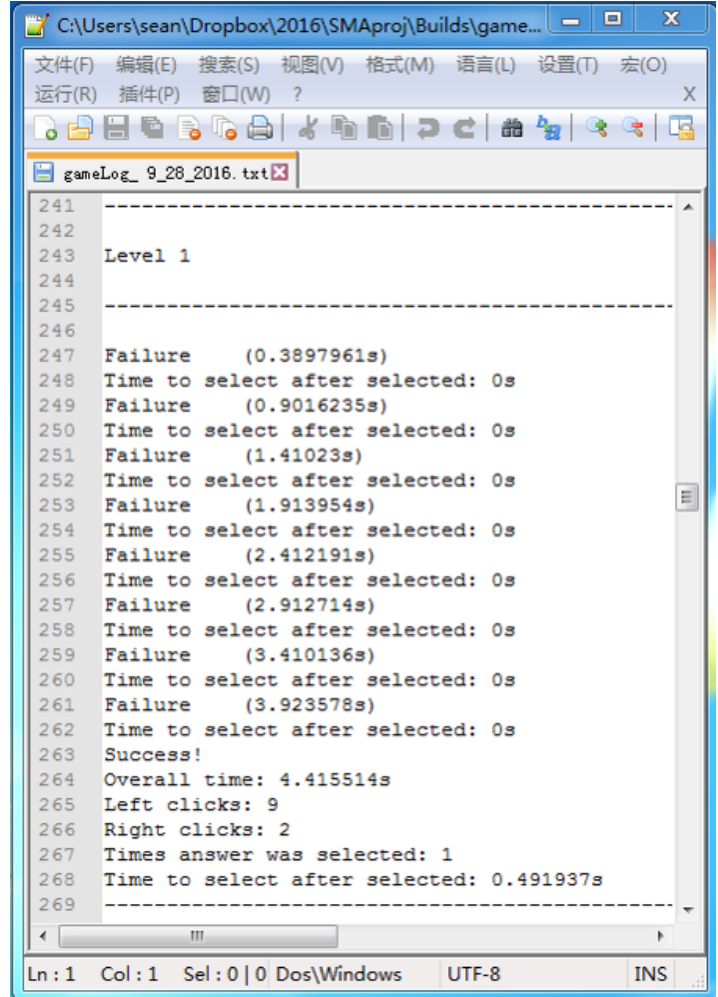

Figure 6. Data tracking

their left hands better while others are good with their left ankles. Therefore, we need to observe participants play with toys, watch videos, and communicate with parents in this phase and then identifying the strongest muscles. Moreover, interviewing parents can also increase the accuracy of result because they are very familiar with the preferences and habits of participants. For example, some children with SMA have both strong muscles on their legs and wrists but they may not be able to keep their wrists on a stable switch. In this case, sensors for leg movement could perform better.

4.3.2. Second phase When we are fully aware of the functionality of participants and their preferences, implementation and introduction of the game based interactive system are the next phase. First, we need to deploy one or multiple sensors and switches for the user and guide him/her to trigger interaction based on information collected from first phase. Several adjustments are required during this phase to make participants comfortable and convenience to use sensor inputs. Furthermore, the position and mounting method should be considered in safety aspect since our targeted users are young children.

After sensor implementation, the next task in this 
phase involves user-guided learning. We need to introduce the interactive game and help users understand the objective of the game. Based on preference information gathered in the first phase, we can set the favorite cartoon character as a goal in each level such as Minions, and then adjust the game difficulty based on users functionality.

4.3.3. Third phase During this phase, the user keeps practicing the interactive game using interactive system for two weeks. Besides the participant practicing the interactive game, parents will also take part in answering a questionnaire at the end. Their feedbacks are extremely critical and helpful since they understand their children very well and can provide an evaluation of the interaction experiences. Moreover, parents will also report potential weaknesses of the system and provide valuable advice. All these feedbacks will be helpful to improve the interactive system, adjust the user study, and collect valuable data in the future.

4.3.4. Pilot study preliminary result The pilot study has been carried out for our SMA participant. His functionalities, preferences, and requirements are collected in the first phase. Then all four kinds of wearable interactive devices are introduced to our user. We have demonstrated the selection game to him, his caregivers and parents. The game and devices are then left for him to use for two weeks. However, because of our users physical conditions, he cannot complete two weeks usage continuously, and he can only play our game and devices when certain conditions are satisfied. Therefore, our users third phase study is still ongoing. Based on parents feedback, the most suitable device for our participant is micro-light sensor based one. But it is worth noticing this result depends on users specific physical conditions and personal preferences. For example, our participants parents also have mentioned that other devices also work well but their boy likes the micro-light device better.

Questionnaires have been distributed to caregivers and parents already and after data collection is completed, we will start analysis in detail.

\section{Conclusions}

This paper introduces a multi-modal communication approach for a special group of users with SMA. Four different kind of reliable and low-cost switches and sensors are adopted in our interactive system to provide a way to interact with computer systems for young children. We have designed a pilot user study for a participant who is three-year old and has type I SMA. The participant is currently conducting the pilot study which includes three phases. In the future, we will include more participants in different ages and functionality and perform further user study. Moreover, a comprehensive assistive healthcare system will be designed to help increasing quality of life for children with SMA.

\section{References}

[1] M. R. Lunn and C. H. Wang, "Spinal muscular atrophy," The Lancet, vol. 371, no. 9630, pp. 2120-2133, 2008.

[2] "What is spinal muscular atrophy?." https: //www.mda.org/disease/ spinal-muscular-atrophy, 2017.

[3] J. Pearn, "Classification of spinal muscular atrophies," The Lancet, vol. 315, no. 8174, pp. 919-922, 1980.

[4] S. Ogino, D. G. Leonard, H. Rennert, W. J. Ewens, and R. B. Wilson, "Genetic risk assessment in carrier testing for spinal muscular atrophy," American journal of medical genetics, vol. 110, no. 4, pp. 301-307, 2002.

[5] B. Hillebrands and K. Ounadjela, Spin dynamics in confined magnetic structures I, vol. 83. Springer Science \& Business Media, 2003.

[6] E. W. Ottesen, "Iss-n1 makes the first fda-approved drug for spinal muscular atrophy," Translational Neuroscience, vol. 8, no. 1, pp. 1-6, 2017.

[7] "The cost of biogens new drug: $\$ 750,000$ per patient." http://www.cbsnews.com/news / the-cost-of-biogens-new-drug-spinraza/ 750000-per-patient/, 2017.

[8] E. P. Armstrong, D. C. Malone, W.-S. Yeh, G. J. Dahl, R. L. Lee, and N. Sicignano, "The economic burden of spinal muscular atrophy," Journal of medical economics, vol. 19 , no. 8, pp. 822-826, 2016.

[9] "Werdnig-hoffmann disease." https:// rarediseases.org/rare-diseases/ werdnig-hoffmann-disease/, 2016.

[10] Z. Tang, J. Guo, S. Miao, S. Acharya, and J. H. Feng, "Ambient intelligence based context-aware assistive system to improve independence for people with autism spectrum disorder," in System Sciences (HICSS), 2016 49th Hawaii International Conference on, pp. 3339-3348, IEEE, 2016.

[11] H. Sampath, R. Agarwal, and B. Indurkhya, "Assistive technology for children with autism-lessons for interaction design," in Proceedings of the 11th Asia Pacific Conference on Computer Human Interaction, pp. 325-333, ACM, 2013.

[12] C. Putnam and L. Chong, "Software and technologies designed for people with autism: what do users want?," in Proceedings of the 10th international ACM SIGACCESS conference on Computers and accessibility, pp. 3-10, ACM, 2008.

[13] C. Guimaraes, M. H. Pereira, and S. Fernandes, "A framework to inform design of learning objects for teaching written portuguese (2nd language) to deaf children via sign language (1st language)," in System Sciences (HICSS), 2015 48th Hawaii International Conference on, pp. 2-10, IEEE, 2015. 
[14] A. Mihailidis and G. R. Fernie, "Context-aware assistive devices for older adults with dementia," Gerontechnology, vol. 2, no. 2, pp. 173-188, 2002.

[15] A. Von Gontard, K. Zerres, M. Backes, C. Laufersweiler-Plass, C. Wendland, P. Melchers, G. Lehmkuhl, and S. Rudnik-Schöneborn, "Intelligence and cognitive function in children and adolescents with spinal muscular atrophy," Neuromuscular Disorders, vol. 12, no. 2, pp. 130-136, 2002.

[16] A. M. Cook and J. M. Polgar, Assistive technologies: Principles and practice. Elsevier Health Sciences, 2014.

[17] I. S. Mackenzie and T. Felzer, "Sak: Scanning ambiguous keyboard for efficient one-key text entry," ACM Transactions on Computer-Human Interaction (TOCHI), vol. 17, no. 3, p. 11, 2010.

[18] M. Zimmerling, F. Ferrari, R. Lim, O. Saukh, F. Sutton, R. Da Forno, R. S. Schmidt, and M. A. Wyss, "A reliable wireless nurse call system: Overview and pilot results from a summer camp for teenagers with duchenne muscular dystrophy," in Proceedings of the 11th ACM Conference on Embedded Networked Sensor Systems, p. 35, ACM, 2013.

[19] T. Guerreiro, H. Nicolau, J. Jorge, and D. Gonçalves, "Towards accessible touch interfaces," in Proceedings of the 12th international ACM SIGACCESS conference on Computers and accessibility, pp. 19-26, ACM, 2010.

[20] S. Trewin, C. Swart, and D. Pettick, "Physical accessibility of touchscreen smartphones," in Proceedings of the 15th International ACM SIGACCESS Conference on Computers and Accessibility, p. 19, ACM, 2013.

[21] P. Carrington, A. Hurst, and S. K. Kane, "The gest-rest: a pressure-sensitive chairable input pad for power wheelchair armrests," in Proceedings of the 16th international ACM SIGACCESS conference on Computers \& accessibility, pp. 201-208, ACM, 2014.

[22] A. Fattouh, M. Sahnoun, and G. Bourhis, "Force feedback joystick control of a powered wheelchair: Preliminary study," in Systems, Man and Cybernetics, 2004 IEEE International Conference on, vol. 3, pp. 2640-2645, IEEE, 2004.

[23] A. Duchowski, Eye tracking methodology: Theory and practice, vol. 373. Springer Science \& Business Media, 2007.

[24] R. Jacob and K. S. Karn, "Eye tracking in human-computer interaction and usability research: Ready to deliver the promises," Mind, vol. 2, no. 3, p. 4, 2003. 